Año LI. urtea

$127-2019$

Urtarrila-ekaina

Enero-junio

5

รar.

ats

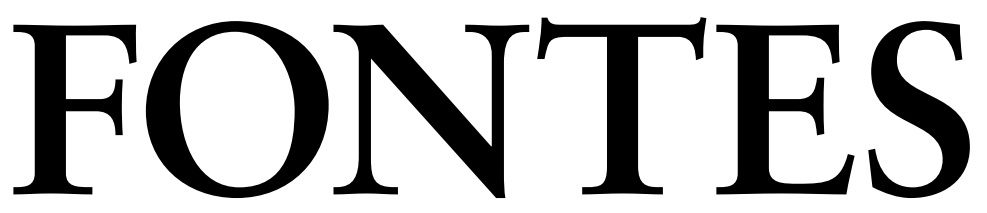

LINGVÆ

VASCONVM

STVDIA ET DOCVMENTA

SEPARATA

\title{
Variantes del topónimo \\ Iruña (siglos XI y XII)
}

Miguel Larrambebere Zabala

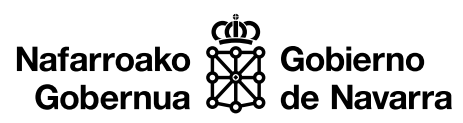

- 00 


\section{Variantes del topónimo Iruña (siglos XI y XII)}

Iruña toponimoaren aldaerak (XI eta XII. mendeak)

Variations of the topomym Iruña (11th and 12th centuries)

Miguel LARRAmbebere Zabala

Seminario Conciliar de San Miguel

Centro Superior de Estudios Teológicos de Pamplona

larrambeapeza@yahoo.es 


\section{RESUMEN}

Iruña es la denominación ancestral que la lengua vasca ha utilizado hasta nuestros días para designar a la ciudad de Pamplona. Menos conocido es el hecho de que este topónimo fue incorporado entre mediados del s. XI y mediados del s. XII a la documentación del reino de Pamplona, especialmente a la emanada de la escribanía regia, para referirse a la sede episcopal y al señorío del obispo sobre la ciudad. Llama la atención que en los documentos del período citado la palabra Iruña presenta diversas variantes: fundamentalmente Eruña, Oruña y Uruña. La procedencia geográfica de los escribas que más usaron dichas formas puede dar luz acerca de las comarcas en que estas tuvieron vigencia.

Palabras clave: topónimo Iruña; Eruña; Oruña; Uruña; Pamplona.

\section{LABURPENA}

Iruña da euskaraz Nafarroako hiriburuari oso aspalditik eman zaion izena. Izen honek bizirik iraun du etenik gabe gaur egun arte euskaran eta hiriaren nahiz inguruko herrien toponimia txikian. Ez da hain ezaguna, aitzitik, herri hizkeran erabiltzen den toponimo hori mende batean zehar, XI. mende erditik XII. mende erdira arte, Iruñeko erresumaren izkribuetan txertatua izan zela; bereziki errege eskribautzan jatorria zuten dokumentuetan apezpiku egoitza eta apezpikuaren jauntasuna aipatzeko. Deigarria da garai horretako dokumentuetan Iruña hitzak hainbat aldaera agertzen dituela: nagusiki, Eruña, Oruña, Uruña. Forma hauek gehien erabili zituzten eskribauen jatorri geografikoak aldaera horien erabilera eremuen berri eman dezake.

Gako hitzak: Iruña toponimoa; Eruña; Oruña; Uruña; Pamplona.

\section{ABSTRACT}

Iruña is the ancient name that the Basque language has used until today to refer to the city of Pamplona. Less well known is the fact that this place name has been incorporated from the middle of the 11th century to the middle of the 12th century into the documents of the kingdom of Pamplona, specially into them that came from the royal clerkship, in order to designate the bishop's seat and the bishop's lordship on the city. It is noteworthy that in the documents of the mentioned period the word Iruña has several variations: mainly Eruña, Oruña, Uruña. The geographical origin of scribes who used these forms can shed light on the counties where they were in effect.

Keywords: Iruña toponym; Eruña; Oruña; Uruña; Pamplona. 
1. PAMPLONA E IRUÑA EN LA DOCUMENTACIÓN DE LA MONARQUÍA PAMPLONESA. 2. VARIANTES DE IRUÑA. 2.1. Eruña. 2.2. Oruña. 2.3. Uruña. 3. USOS DE CANCILleRÍA Y PROCEDENCIA GEOGRÁFICA DE LOS ESCRIBAS. 4. BREVE ALUSIÓN A LAS OTRAS "IRUÑAS". 5. EPÍlOGO. 6. REFERENCIAS.

\section{PAMPLONA E IRUÑA EN LA DOCUMENTACIÓN DE LA MONARQUÍA PAMPLONESA}

Iruña es la denominación ancestral que la lengua vasca ha usado para referirse a la ciudad de Pamplona. Presente en los textos literarios de los autores vascos clásicos, ha perdurado hasta nuestros días sin solución de continuidad en el vascuence y en la toponimia menor de la misma ciudad y de los pueblos de su entorno, la Cuenca o Tierra de Pamplona, Iruñerria ${ }^{1}$.

Menos conocido es el hecho de que este topónimo fue incorporado a lo largo de un siglo, entre mediados del s. XI y mediados del s. XII, a la documentación del reino pirenaico, especialmente a la emanada de la escribanía regia ${ }^{2}$. Este hecho se inscribe en el contexto de la progresiva definición de los espacios de soberanía real y de su distinción con respecto al estatus jurídico de la antigua civitas pamplonesa, sede del obispo, que además era señor temporal de la ciudad. En efecto, fue en el reinado de García Sánchez III, en torno al año 1047, cuando Iruña hizo su aparición en los diplomas reales

1 Cfr. Jimeno Jurío (1986, p. 172) y, más ampliamente, en los exhaustivos repertorios que el mismo autor publicó (1986-1992). En la propia ciudad de Pamplona existen los términos de Irunlarrea e Iruñeuntze, equivalentes ambos a Prado o Soto de Pamplona (Jimeno Jurío \& Salaberri, 1994, pp. 262-264). Se podría advertir la difusión del nombre romance en sustitución del tradicional en topónimos relativamente modernos como Pamplonagain, Pamplonagañeko Treguarria, Pamplonako Harrate, Pamplonako Zoko en Uharte Arakil (Jimeno Jurío, 1993, p. 159).

2 Cfr. para toda esta cuestión Larrambebere (1998, pp. 2-27). 
con el fin de designar la ciudad episcopal y a la sede del obispo (Santa María de Iruña), mientras se tendía a reservar el nombre de Pamplona para aludir al reino. En el reinado de Sancho Garcés IV esta iniciativa fue ganando terreno y se implantó con fuerza en tiempos de Pedro I y Alfonso I el Batallador, después de un momento de vacilación con Sancho Ramírez. El reinado de García Ramírez aporta los últimos ejemplos de la presencia de Iruña en los diplomas regios pamploneses (1140-1141). Contemporáneamente a estos últimos casos, los escribas que Ramiro II el Monje heredó de su hermano Alfonso I continuaron con esta tradición en los diplomas del ámbito aragonés.

Las escribanías de las instituciones eclesiásticas asumieron con poco entusiasmo esta innovación en los usos cancillerescos. Sólo en el monasterio de Leire, cenobio estrechamente ligado a los dinastas pamploneses, tuvo cierto arraigo, mientras que fue menor su acogida en otros centros religiosos. La catedral de Pamplona, el establecimiento más afectado por el cambio, se aferró casi siempre a la denominación de sede pamplonesa y Santa María de Pamplona, especialmente en los documentos directamente vinculados a la mitra.

Con la adopción de Navarra como nombre definitivo del reino en tiempos de Sancho VI el Sabio (1162) se superó la problemática polisemia de Pamplona, que en adelante se reservó para denominar a la sede y a la ciudad gobernada por el obispo.

\section{VARIANTES DE IRUÑA}

Según se ha señalado, es, sin duda, Iruña $a^{3}$ la denominación vernácula que más habitualmente aparece en los diplomas del período mencionado para referirse a la sede episcopal y a la ciudad de Pamplona. Sin embargo, conviene tener en cuenta que los textos muestran una notable riqueza de variantes en el uso de dicho topónimo, aspecto al que prestaremos atención en este artículo.

Aparte de los errores atribuibles a los copistas ${ }^{4}$ y de las diferencias en la grafía ${ }^{5}$, está la forma Iroña, que se constata en 27 documentos privados del monasterio de Leire, elaborados entre 1083 y $1115^{6}$, y en algunos recogidos en el Libro Redondo de la

3 En la documentación medieval la palatalización de la «n» se representa de muy diversas maneras. Así, no es raro encontrar en ella las grafías Iruina, Iruinia, Irunga, Irunia, Irunna, Irunnia, Irunya, Iruyna, y otras, todas las cuales deben leerse Iruña. Policarpo de Iraizotz (1980, p. 428, n. 7) recoge un ejemplo análogo en las distintas variantes del actual topónimo Marañón. Nótese, además, que la adopción de la grafía Iruña en este contexto, como transcripción de la grafía latina, no tiene implicación alguna en la cuestión relativa al nombre vasco de Pamplona: $c f r$. para esto último lo señalado por Michelena (1983, pp. 445-451), así como el comentario de Knörr (1990, p. 127) y la esclarecedora recapitulación de Salaberri (1993, pp. 167-192).

4 Así, por ejemplo, la voz Irunça (Martín Duque, 1983, p. 153).

5 Además de la voz citada en la nota anterior, se ha encontrado la grafía Hirunia en contexto documental riojano (Rodríguez de Lama, 1976, n. ${ }^{\circ} 32$ ).

6 Martín Duque, 1983, n. ${ }^{\text {os }} 110,124,125,147,151,152,156,172,174,193,194,196,197,201,203,208$, 210, 217, 220, 224, 226-228, 231, 234, 241, 256. Reparó en ello Jimeno Jurío (1991, p. 65). 
catedral de Pamplona ${ }^{7}$. En algún caso se documenta también la grafía Irunea ${ }^{8}$ o Irunie ${ }^{9}$. En escasos textos el topónimo en cuestión pierde la vocal inicial y se convierte en Runia o Ruina, variante que ha sido relacionada con el hidrónimo Runa, correspondiente al más importante de los cursos fluviales que bañan el término pamplonés ${ }^{10}$.

\subsection{Eruña}

Es ésta una variante relativamente frecuente en los diplomas regios del período que aquí se contempla. Hallamos los ejemplos más antiguos en sendos documentos de García de Nájera y Sancho de Peñalén conservados en el entorno geográfico najerense ${ }^{11}$. El escriba real García (probablemente, el apellidado de Biel) la anotó en cuatro documentos de Sancho Ramírez (abril y mayo de 1085) 12 $^{12}$ Pero es en la escribanía de Pedro I donde realmente se prodiga el uso de Eruña: 21 ejemplos de entre 70 referencias documentales que aluden al nombre popular de la sede pamplonesa ${ }^{13}$. Dichos ejemplos están incluidos en textos redactados por algunos de los escribas reales más activos del momento: Galindo, García -quizá uno de los que con este nombre trabajó al servicio de Sancho Ramírez- y Sancho, nombre que se repite acompañado de distintos determinantes (scriptor, Garcez, de Iesero) y que no es seguro corresponda a la misma persona ${ }^{14}$. Entre los escribas de Alfonso I el Batallador decae la utilización de la variante: sólo 6 referencias ${ }^{15}$ entre un total de 113 alusiones al topónimo. En este caso, las menciones se deben a la pluma de Sancho de Perarrúa, Eneco y de un Sancho que no es seguro corresponda al anterior ${ }^{16}$.

7 Goñi, 1997, 22 (1070); 37 (1085); 440 (s. XII).

8 Martín Duque, 1983, n. 313 (año 1139): uilla Yrunea, referido en este caso no a Pamplona, sino a Iruñela (Yerri). Otro ejemplo en una versión de la donación del fuero de Calahorra a los pobladores de Funes, Marcilla y Peñalén, publicada por Muñoz (1847, p. 428): Episcopus Petrus in Irunea. Sin embargo, la versión ofrecida por Lema (1990, n. $\left.{ }^{\circ} 41\right)$, que lo fecha en 1110, dice Irunnia.

9 Goñi, 1997, n. ${ }^{\circ} 22$ (1070). Esta forma en su caso genitivo locativo se documenta en el nombre de un testigo que en un mismo texto aparece mencionado de dos modos distintos: senior Lope Enecones de Irunia y senior Enecones Yrunieco (Goñi, 1997, n. ${ }^{\circ}$ 140, año 1122).

10 Cfr. Jimeno Jurío, 1993, pp. 507-520, especialmente pp. 514-518. Las citas documentales en que se basa están insertas en sendas concesiones forales de 1102 (Pedro I a los pobladores de Caparroso) y 1129 (Alfonso I a los francos de San Saturnino de Iruña): Ubieto, 1951, n. ${ }^{\circ} 114$; Molho, 1963, p. 291. Esta última está incluida en una versión en romance navarro-aragonés, preparada en fecha anterior a 1340, probablemente en Villafranca de Navarra. En el texto recogido por Molho, la concesión foral se dirige a los «ffrancs que poblareç en aquela plana de Ssanct Cerni de Ruina», pero en la cláusula Regnante del mismo se lee «Sancho, hobispo en Yruina» (edición posterior en Lacarra, Martín Duque, 1975, pp. 117-123). A estos dos testimonios que trae a colación José M $\mathrm{M}^{\mathrm{a}}$ Jimeno Jurío, cabría añadir otros dos diplomas regios de 1115 y 1133 (Lema, 1990, n. ${ }^{\circ} 62$ y 270), este último conservado en copia del siglo XVI con abundantes errores de lectura. Por otra parte, se ha detectado en una ocasión un Irrunia, forma que, al igual que los casos comentados, presenta vibrante fuerte, pero aquí conserva además la vocal inicial (Canellas, 1993, n. ${ }^{\circ} 85$, original, según el autor de la colección).

11 Ubieto, 1976, n. ${ }^{\text {os }} 222$ (1042?), 396 (1072).

12 Salarrullana, 1907, pp. 25-27; Canellas, 1993, n. 77.

13 Ubieto, 1951, n. os 38, 45, 48, 49, 54, 57, 59, 65, 66, 72, 73, 75, 77, 79 (versión B) 103, 116, 123, 130, 131, 139. Además, el rescatado por García Larragueta (1971, pp. 47-56).

14 Canellas, 1983, p. 27; Lema, 1990, pp. 169-170.

15 Lema, 1990, n. ${ }^{\text {ss }} 5,71,126,194,195,201$ (versión B).

16 Canellas, 1983, p. 28; Lema, 1990, pp. XI-XII. 


\subsection{Oruña}

De uso menos frecuente es la segunda forma que se ha considerado en este elenco de variantes: Oruña. Un ejemplo en la documentación de Sancho Ramírez ${ }^{17}$, dos en la de Pedro ${ }^{18}$, tres en la de Alfonso $I^{19}$, uno en la de García Ramírez ${ }^{20}$ y otro en la de Ramiro II $^{21}$ la han conservado. Casi todos estos diplomas -en el del Restaurador no consta data tópica-fueron expedidos en ámbitos apartados del núcleo propiamente pamplonés: San Juan, San Pedro de Taberna, Calasanz, Tardajos, Cantabria (frente a Logroño), Fraga y Uncastillo. El primero de los casos de época de Pedro I fue redactado por Juan de Olsón $^{22}$, que no formaba parte del habitual equipo de escribas regios, y que, por lo tanto, actuó excepcionalmente al servicio del monarca. El segundo lleva la suscripción de un Sancho, pero se trata de un documento falso. Los mencionados textos del Batallador fueron elaborados por Galindo, Bernardo de Jaca y Miguel ${ }^{23}$, también escribas ocasionales. El ejemplo incluido en un documento del Restaurador no presenta la correspondiente suscripción del escriba. Por último, el de Ramiro II fue redactado por un Sancho, tal vez el de Perarrúa. Hay también dos documentos privados de Santa María de Uncastillo ${ }^{24}$ y otros dos, expedidos, al parecer, en Borja ${ }^{25}$ que presentan la voz Oruña.

Las variantes que acaban de ser analizadas, Eruña y Oruña, son desconocidas en los diversos dialectos vascos existentes en la actualidad y tampoco se ha podido hallar testimonio alguno de su uso en las últimas centurias ${ }^{26}$.

\subsection{Uruña}

Toca ahora aludir a la forma Uruña, que aflora en 8 diplomas de Pedro ${ }^{27}$ (redactados por los escribas Galindo, Sancho, otro Sancho, García y Juan, capellán de la reina Berta), 13 de su hermano y sucesor Alfonso ${ }^{28}$ (Sancho, García, Sancho de Perarrúa, Eneco, Pedro -capellán de Ato Orella-, Aznar y Domingo) y otros 5 de Ramiro II el

17 Durán, 1965, n. $^{\circ} 40$.

18 Lema, 1990, n. ${ }^{\text {os }} 61,62$ (falso).

19 Lema, 1990, n. ${ }^{\text {s }} 103,249,258$.

20 Lacarra, 1982, n. ${ }^{\circ} 294$.

21 Ubieto, 1988, n. $^{\circ} 92$.

22 Canellas, 1983, pp. 27-28; Lema, 1990, n. ${ }^{\text {s }} 168-169$.

23 Canellas, 1983, pp. 27-28; Lema, 1990, pp. XI-XII.

24 Martín Duque, 1962, n. ${ }^{\circ} 2$ (1100), 46 (ca. 1142-1159).

25 Lacarra, 1982, n. 310 (1141), repetido en Lacarra, 1985, n. 325 (1144?).

26 Sota, Lafitte y Akesolo (1980, p. 2227; 1989, p. 3730) recogen ejemplos del uso de Iruña en algunos textos vascos de los siglos XVII-XVIII y reproducen en la voz Uruña lo que Azkue ya escribió en su diccionario ( $c f r$. nota 32 de este artículo). Sin embargo, no aluden para nada a las formas Eruña y Oruña. Tampoco lo hace el monumental Diccionario General Vasco de Michelena (1987-2005, 16 vols.).

27 Ubieto, 1951, n. ${ }^{\text {ss }} 15,20,63,100,107,120$ (falso), 125, 149.

28 Lema, 1990, n.os 9, 21, 111 (versión C), 153, 167, 170, 176, 196, 199, 239, 263, 268, 279, 281. 
Monje $^{29}$ (Eneco y Sancho de Perarrúa). Entre la documentación emanada de los establecimientos eclesiásticos hay que traer a colación en este apartado un diploma de San Juan de la Peña ${ }^{30}$ y otro de la catedral de Huesca ${ }^{31}$.

Al contrario de lo referido con respecto a otras variantes ya citadas, es preciso señalar que el término Uruña ha sido empleado hasta tiempos recientes para designar la capital navarra por los hablantes del dialecto roncalés en su variedad de Uztárroz ${ }^{32}$.

\section{USOS DE CANCILLERÍA Y PROCEDENCIA GEOGRÁFICA DE LOS ESCRIBAS}

A pesar de los problemas que plantea la identificación de los escribas (García Larragueta, 1983, p. 19) y sin perder de vista la prudencia que requiere cualquier hipótesis basada en textos de tan borrascosa tradición documental, una aproximación a la procedencia de los funcionarios que hicieron uso de las variantes Eruña, Oruña y Uruña puede sugerir el ámbito geográfico en que dichas formas tuvieron vigencia.

Entre los nombres de los escribas que interesan al caso sólo unos pocos explicitan con meridiana claridad su origen geográfico: García [de Biel?], Sancho de Yésero, Sancho de Perarrúa, Juan de Olsón y Bernardo de Jaca. Estos topónimos remiten a poblaciones de Aragón (Jaca, Yésero y Biel), Sobrarbe (Olsón) y Ribagorza (Perarrúa), patria de buena parte de los amanuenses que trabajaron al servicio de los monarcas de Pamplona y Aragón ${ }^{33}$. En el resto de los casos no se han conservado los determinantes geográficos, si bien algunos nombres pueden corresponder también al espacio aragonés -es el caso de los escribas denominados Galindo y Aznar-, mientras que otros son antropónimos, tanto de uso local (García, Eneco, Sancho) como universal (Miguel, Juan, Pedro, Domingo), que no permiten mayores concreciones.

En cuanto a los diplomas privados, sólo las emplean unos pocos procedentes de San Juan de la Peña, la catedral de Huesca y Santa María de Uncastillo.

29 Ubieto, 1988, n. ${ }^{\circ} 5,63,65,67,68$.

30 Ubieto, 1963, n. $^{\circ} 154(1060)$.

31 Durán, 1965, n. ${ }^{\circ} 112$ (1113).

$32 \mathrm{La}$ forma empleada por los vascoparlantes roncaleses de las otras localidades del valle era Uriña (Michelena, 1958, pp. 35-47). Interesan también los comentarios al respecto de Salaberri (1993, p. 188). Resurrección M. ${ }^{a}$ de Azkue (1906, p. 381) aventuró la opinión de que Uruña era el nombre usual de Pamplona en la Edad Media y no Iruña, que se habría extendido más tarde. Es posible que este sorprendente aserto tenga que ver con una idea de Arturo Campión (1929, pp. 47-48), para quien la variante Uruña conservaría mejor que Iruña el primer componente de la palabra.

33 Es importante tener en cuenta que «por su posición central, es el altoaragonés el más frecuentado de los tres espacios históricos de la monarquía; dentro de sus límites se fechó el $23 \%$ de los diplomas de Pedro I y el $31 \%$ de los de Alfonso I. Los porcentajes descienden, respectivamente, al $18 \%$ y el $5 \%$ para SobrarbeRibagorza y al 16\% y el 7\% para el territorio navarro» (Martín Duque, 1992, p. 254). 
En algunas copias de documentos realizadas en el ámbito aragonés se percibe la sustitución de una variante por otra: así, Iruña se convierte en Eruña en dos copias del monasterio de Jesús Nazareno de Montearagón ${ }^{34}$, realizadas en el siglo XIII, y en Urruña en una versión conservada en el Archivo Municipal de la localidad jacetana de Araguás del Solano ${ }^{35}$, mientras que Oruña pasa a ser Uruña en copia de una confirmación de Jaime II (1322) custodiada en el Archivo de la Corona de Aragón ${ }^{36}$. En este mismo archivo existe una copia en la que el primitivo Uruña fue sustituido por el más común Iruña $a^{37}$.

Estos datos parecen converger, en líneas generales, en la consideración de los territorios orientales de la monarquía (Aragón, Sobrarbe, Ribagorza) como circunscripciones en las que se usaron las citadas variantes. Por otra parte, interesa resaltar que en la documentación real pamplonesa es mínimo el uso de las variantes citadas antes de la unión de los reinos de Aragón y Pamplona.

Cabría hacer aquí otras consideraciones que entrarían ya en el campo de la sociolingüística, como la circunstancia de que estos escribas aragoneses que emplean esporádicamente las variantes estudiadas utilizan, sin embargo, con mucha mayor frecuencia la versión Iruña, más extendida en las tierras estrictamente pamplonesas y arraigada desde tiempo atrás en la escribanía regia. No hay que olvidar tampoco que algunos escribas usan al mismo tiempo distintas variantes: es el caso de Sancho de Perarrúa, entre cuyos documentos se puede hallar tanto alusiones a Iruña, Eruña y Uruña como a Pamplona ${ }^{38}$. Hay también amanuenses que prefieren escribir siempre el topónimo Pamplona, como hace el jacetano Sancho de Larrosa ${ }^{39}$, canónigo de Huesca y posteriormente obispo de la sede iruñense.

\section{BREVE ALUSIÓN A LAS OTRAS «IRUÑAS»}

No está de más incluir en este panorama las variantes de la Iruña de Yerri (Iruñela) y la situada en la Tierra de Nájera (Uruñuela). En cuanto a la primera, hay que decir que

34 Ubieto, 1951, n. ${ }^{\circ} 79$; Lema, 1990, n. 201.

35 Lema, 1990, n. ${ }^{\circ}$ 111. Este diploma, expedido en el valle de Soule (Zuberoa) y elaborado por el caballero Pedro Bernardo de Fantova, plantea una interesante cuestión. Según el ejemplar A, establecido por José Angel Lema Pueyo, la versión original de la cláusula Regnante diría Sancius episcopus in Irunia, al igual que la versión B, copia del siglo XIII conservada en el Archivo Municipal de Araguás del Solano. Por el contrario, otra copia -ejemplar C-, inserta en una confirmación de Juan I (6 de junio de 1389) y custodiada en el mismo archivo, recoge la expresión Sancius episcopus in Urrunia. Comentarios sobre el vasallaje de este caballero en Martín Duque, 1992, p. 392, n. 35.

36 Lema, 1990, n. 249.

37 Lema, 1990, n. 9.

38 Cfr., por ejemplo, Lema, 1990, n. ${ }^{\text {s }} 41,71,167$ y 229.

39 Cfr., por ejemplo, Ubieto, 1951, n. ${ }^{\text {os }} 46,147,150$. 
en los documentos más antiguos aparece en la forma más común de Iruña $a^{40}$. Sólo en algunos textos más tardíos (siglos XIII-XIV) se la identifica por excepción como Eruña ${ }^{41}$ o Eruñela ${ }^{42}$. Por el contrario, la Uruña de la Tierra de Nájera, actual Uruñuela, presenta desde un principio formas variadísimas: Iruña ${ }^{43}$, Eruña / Eruñuela ${ }^{44}$, Oruña / Oruñue$l a^{45}$, Uruña / Uruñuela ${ }^{46}$. En una sola ocasión se ha podido encontrar una referencia a la que parece ser la Iruña de Alava, citada como Oruña ${ }^{47}$.

\section{EPÍlOGO}

Los análisis documentales y toponímicos confirman que, en efecto, la forma Iruña fue en el habla popular la más habitualmente empleada en el territorio originariamente pamplonés para designar la caput regni y su homónima de Yerri. De ahí que sea la variante con mayor presencia en los diplomas emanados de la escribanía regia cuando se hace uso de este nombre para aludir a la civitas y sede episcopal de Pamplona.

Las variantes Eruña, Oruña y Uruña, referidas a Pamplona y a otras poblaciones como Iruñela (Yerri) y Uruñuela (Nájera), afloran con diversa frecuencia en la documentación. Tales formas sustituyeron a Iruña o convivieron con este vocablo en el uso de los escribas procedentes de otros sectores menos «nucleares» de la monarquía. Pudiera apuntar este hecho a unas variantes presentes en el habla de los naturales de las comarcas orientales de la monarquía (Roncal, Alto Aragón y Sobrarbe-Ribagorza), de Tierra Estella, así como de la franja suroccidental (Nájera).

40 Martín Duque, 1983, n.os 163 (1098), 313 (1139). De 1195 es la referencia a un Lupus de Irunnuela (García Larragueta, 1957, n. $\left.{ }^{\circ} 77\right)$. Casi medio siglo más tarde, en 1230, se menciona al magister P. de Irunnola (García Larragueta, 1957, n. ${ }^{\circ} 230$ ). A principios del siglo XV vivían en Estella Pascoal de Iruinela y Pero Sanchiz de Iruinela (Arraiza, 1968, pp. 140, 144).

41 El Libro del monedaje de tierras de Estella (1350) menciona a un Miguel Periz Eruinaqua («de Eruña») entre los vecinos de Izurzu (Carrasco, 1973, p. 371). Sin embargo, en el mismo libro, al igual que en el de fuegos de 1366, se encabeza el apartado correspondiente a la villa en cuestión con el título de Yruynela («Iruñela») (Carrasco, 1973, pp. 368, 584). Parece clara la distinción entre una variante de uso coloquial y otra con visos de mayor oficialidad.

42 Lacarra, Martín Duque, 1986, n. ${ }^{\circ} 516$ (1377); Jimeno Jurío, 1970, pp. 221-269 y n. ${ }^{\circ} 114$ (1257).

43 Ubieto, 1981, n. ${ }^{\circ} 12$ (945); Rodríguez de Lama, 1976, n. ${ }^{\circ} 82 \mathrm{e}$ (ca. 1125-1146).

44 Ubieto, 1976, n. ${ }^{\text {os }} 178$ (1024), 293 (1055); Rodríguez de Lama, 1976, n. os 13, 14 (1052-1054), 186 (1155).

45 Martín Duque, 1983, n. 43 (1048); Ubieto, 1976, n. 260 (1049); Rodríguez de Lama, 1976, n. 14 (10521054); Rodríguez de Lama, 1979, n. 305 (1189). El Diccionario de la Real Academia de la Historia (1846, p. 143) todavía introduce el artículo dedicado a esta población riojana por «Oruñuela o Uruñuela».

46 Martín Duque, 1983, n. 34 (1043); Ubieto, 1976, n. ${ }^{\text {s }} 268$ (1050), 293 (1055), 321 (1062).

47 Lema, 1990, n. ${ }^{\circ}$ 103. El escriba Galindo presenta en este documento al obispo Guillermo in Oruna. Pero, además de esta inevitable alusión al prelado pamplonés, incluye en la relación de tenentes, entre los seniores de Calahorra, Nájera, Cerezo y Belorado, a un Calvet Juanes in Oruna. El contexto permite suponer que se trata de la ya mencionada Iruña alavesa, que habría adquirido la categoría de tenencia en esta marca suroccidental recuperada por Alfonso I el Batallador. Es interesante constatar que el redactor del diploma reproduce para el caso alavés la variante que ha utilizado al referirse a la sede de Pamplona. 


\section{REFERENCIAS}

Arraiza Frauca, J. (1968). Los fuegos de la merindad de Estella en 1427. Príncipe de Viana, 29, 117-147.

Azkue Aberasturi, R. Ma de (1906). Diccionario vasco-español-francés. Bilbao: El autor.

Campión Jaimebón, A. (1929). Nabarra en su vida histórica. Pamplona: Euskal-Erriaren alde.

Canellas López, Á. (1983). La cancillería real del reino de Aragón (1035-1134). En Folia Budapestina (pp. 23-46). Zaragoza: Institución Fernando el Católico.

Canellas López, Á. (1993). Colección diplomática de Sancho Ramírez. Zaragoza: Real Sociedad Económica Aragonesa de Amigos del País.

Carrasco Pérez, J. (1973). La población de Navarra en el siglo XIV. Pamplona: Eunsa.

Durán Gudiol, A. (1965). Colección diplomática de la catedral de Huesca (vol. 1). Zaragoza: Instituto de Estudios Pirenaicos.

García Larragueta, S. (1957). El gran priorado de Navarra de la Orden de San Juan de Jerusalén. Siglos XII-XIII (vol. 2). Pamplona: Institución Príncipe de Viana.

García Larragueta, S. (1971). Un documento original e inédito de Pedro I. En Homenaje a don José Esteban Uranga (pp. 47-56). Pamplona: Aranzadi.

García Larragueta, S. (1983). Los documentos de los reyes de Pamplona en el siglo XI. En Folia Budapestina (pp. 7-22). Zaragoza: Institución Fernando el Católico.

Goñi Gaztambide, J. (1997). Colección diplomática de la Catedral de Pamplona. Tomo I (829-1243). Pamplona: Institución Príncipe de Viana.

Jimeno Jurío, J. M. (1970). El Libro Rubro de Iranzu. Príncipe de Viana, 31, 221-269.

Jimeno Jurío, J. M. ${ }^{a}$ (1986). Nombres vascongados y romanceados de pueblos navarros. Fontes Linguae Vasconum, 47, 171-182.

Jimeno Jurío, J. M. ${ }^{a}$ (1986-1992). Toponimia de la Cuenca de Pamplona: Cizur, Galar, Olza, Iza, Ansoain. Bilbao: Euskaltzaindia. (Onomasticon Vasconiae, vols. 1, 2, 3, 6, 9).

Jimeno Jurío, J. M. ${ }^{a}$ (1991). Pamplona y sus nombres. Fontes Linguae Vasconum, 57, $55-76$.

Jimeno Jurío, J. M. (1993). Arga et Runa flumen. Fontes Linguae Vasconum, 64, 507520.

Jimeno Jurío, J. M. a (1993). Nafarroako toponimia eta mapagintza. XII. Etxarri Aranatz-Arbizu-Lakuntza-Arruazu-Uharte Arakil-Irañeta-Aralar, Toponimia y cartografía de Navarra. Pamplona: Gobierno de Navarra. Departamento de Educación y Cultura.

Jimeno Jurío, J. M a \& Salaberri Zaratiegi, P. (1994). Toponimia de la Cuenca de Pamplona: Pamplona / Iruña. Bilbao: Euskaltzaindia. (Onomasticon Vasconiae, vol. 12).

Knörr Borrás, E. (1990). Bibliografía. Fontes Linguae Vasconum, 55, 123-130.

Lacarra de Miguel, J. M. ${ }^{a}$ (1982-1985). Documentos para el estudio de la reconquista y repoblación del valle del Ebro (2 vols.). Zaragoza: Anubar.

Lacarra de Miguel, J. M. a \& Martín Duque, Á. J. (1975). Fueros de Navarra. 1. Fueros derivados de Jaca. 2. Pamplona. Pamplona: Institución Príncipe de Viana. 
Lacarra de Miguel, J. M. ${ }^{a}$ \& Martín Duque, Á. J. (1986). Colección diplomática de Irache. 2 (1223-1397). Pamplona: Institución Príncipe de Viana.

Larrambebere Zabala, M. (1998). Pamplona e Iruña (siglos XI y XII). En Navarra y Europa. Tercer Congreso General de Historia de Navarra. Pamplona, 20 23 de septiembre de 1994 (pp. 2-27). Pamplona: Gobierno de Navarra. Departamento de Educación y Cultura.

Lema Pueyo, J. Á. (1990). Colección diplomática de Alfonso I de Aragón y Pamplona (1104-1134). San Sebastián: Eusko Ikaskuntza.

Martín Duque, Á. J. (1962). Cartulario de Santa María de Uncastillo (siglo XII). Estudios de Edad Media de la Corona de Aragón, 7, 647-740.

Martín Duque, Á. J. (1983). Documentación medieval de Leire (siglos IX a XII). Pamplona: Institución Príncipe de Viana.

Martín Duque, Á. J. (1992). Monarcas y cortes itinerantes en el reino de Navarra. En Viajeros, peregrinos, mercaderes en el Occidente medieval. XVIII Semana de Estudios Medievales. Estella, 22-26 de julio de 1991 (pp. 245-270). Pamplona: Institución Príncipe de Viana.

Martín Duque, Á. J. \& Ramírez Vaquero, E. (1992). Aragón y Navarra: instituciones, sociedad, economía (siglos XI y XII). En Historia de España Menéndez Pidal. 10-2. Los reinos cristianos en los siglos XI y XII. Economías, sociedades, instituciones (pp. 337-444). Madrid: Espasa-Calpe.

Michelena Elissalt, L. (1958). Hispánico antiguo y vasco. Archivum, 8, 35-47.

Michelena Elissalt, L. (1983). Iruñea. En Piarres Lafitte-ri omenaldia (pp. 445-451). Bilbao: Euskaltzaindia.

Michelena Elissalt, L. (1987-2005). Diccionario general vasco / Orotariko euskal hiztegia (16 vols.). Bilbao: Euskaltzaindia.

Molho, M. (1963). El fuero de Jaca. Zaragoza: Instituto de Estudios Pirenaicos.

Muñoz Romero, T. (1847). Colección de fueros municipales y cartas pueblas de los reinos de Castilla, León, Corona de Aragón y Navarra (vol. 1). Madrid: Imprenta de Don José María Alonso.

Policarpo de Iráizoz (1980). Nombre vasco de Pamplona: Iruñea, Iruña, Iruñe? Fontes Linguae Vasconum, 35-36, 427-434.

Real Academia de la Historia (1846). Diccionario geográfico-histórico de España (sección 2). Madrid: Imprenta Viuda de Jordán.

Rodríguez de Lama, I. (1976-1979). Colección diplomática medieval de la Rioja (9231225) (vols. 2 y 3). Logroño: Instituto de Estudios Riojanos.

Salaberri Zaratiegi, P. (1993). Nafarroako hiriburuaren izenaren gainean. Euskera, 38 , 167-192.

Salarrullana de Dios, J. (1907). Documentos correspondientes al reinado de Sancho Ramirez, desde MLXIII hasta MLXXXXIIII años. Documentos reales procedentes de la Real Casa y Monasterio de San Juan de la Peña (vol. 1). Zaragoza: Tipógrafo M. Escar.

Sota, M. de la, Lafitte, P. \& Akesolo, L. de (1980 y 1989). Diccionario Retana de Autoridades del Euskera (vols. 5 y 9). Bilbao: La Gran Enciclopedia Vasca.

Ubieto Arteta, A. (1951). Colección diplomática de Pedro I de Aragón y Navarra. Zaragoza: Escuela de Estudios Medievales.

Ubieto Arteta, A. (1963). Cartulario de San Juan de la Peña (vol. 2). Valencia: Anubar. 
Ubieto Arteta, A. (1976). Cartulario de San Millán de la Cogolla (759-1076). Valencia: Anubar.

Ubieto Arteta, A. (1981). Cartulario de Albelda. Zaragoza: Anubar.

Ubieto Arteta, A. (1988). Documentos de Ramiro II de Aragón. Zaragoza: Anubar. 